\title{
A COEFFICIENT PROBLEM FOR FUNCTIONS UNIVALENT IN AN ANNULUS
}

\author{
By Yusaku Komatu
}

\section{Introduction.}

Let $f(z)$ be an analytic function regular and univalent in $z<1$ whose Taylor series expansion is of the form

$$
f(z)=\sum_{n=1}^{\infty} a_{n} z^{n} \quad\left(a_{1}=1\right) .
$$

Coefficient problems for functions univalent in the unit circle have been attacked from various aspects by several authors. Among others, Bieberbach [2] has announced, after proving $a_{2} \leqq 2$, a famous conjecture that there would hold, in general, the estimation

$$
a_{n} \leqq n \quad(n=2,3, \cdots)
$$

accompanied by extremal functions

$$
f^{*}(z)=\frac{z}{(1-\varepsilon z)^{2}}=\sum_{n=1}^{\infty} n \varepsilon^{n-1} z^{n} \quad(\varepsilon=1) .
$$

But this conjecture has been affirmed since then only for $n=3$ by Löwner [11] and for $n=4$ by Garabedian and Schiffer [6], while its general validity seems probable. Bieberbach [3] has derived, in general, only a rough inequality $a_{n}<e^{3} n^{2} / 4$. Littlewood [10] has subsequently succeeded to ameliorate it by proving a more precise inequality

$$
a_{n},<e n
$$

which has been obtained soon after also by Prawitz [13] in a different way. Several sorts of refinement have been brought from various aspects. Detailed results in this field have been collected in a book worked by Schaeffer and Spencer [14]; cf. also a survey written by Bernardi [1] which involves several references.

Compared with a plenty of results on coefficient problems for functions univalent in a circle, very little attention has been given to the corresponding problems for functions univalent in an annulus.

Let $R$ be a real number greater than unity and $w=F(z)$ be a regular analytic function which maps the annulus

Received March 3, 1956. 


$$
1<z^{\prime}<R
$$

univalently onto a ring domain contained in the exterior of the unit circle in such a manner that $z=1$ corresponds to $w=1$, and let its Laurent expansion be

$$
F(z)=\sum_{n=-\infty}^{\infty} c_{n} z^{n} .
$$

We may suppose without loss of generality that a conditon of normalization expressed by

$$
F(1)=1
$$

is to be satisfied, so far as concern the absolute values of the coefficients. The family consisting of all such functions will be designated by $\mathfrak{F}_{R}$. In view of the definition, the family $\mathfrak{F}_{R}$ contains, together with a function $F(z)$, any function $F\left(\varepsilon_{z}\right) e^{-\imath \arg F(\varepsilon)}$ for every $\varepsilon$ with ' $\varepsilon=1$. In particular, if there holds $F(-1)=-1$, then $-F(-z)$ belongs to $\mathfrak{F}_{R}$ provided $F(z)$ so does.

Our problem is then to estimate $c_{n}$ from above, i. e., to obtain an upper bound for $c_{n}$ depending only on $n$ which is valid for any function in $\mathfrak{F}_{R}$. Since the family $\mathfrak{F}_{R}$ is locally bounded ( $\dot{c} f$. the distortion theorem referred to below) and hence normal, the maximum of $c_{n n}$ is really attained for every $n$ by some functions of the family. However, it will be very difficult to solve the extremal problem completely, since even the Biberbach's conjecture is still open. The present paper involves, accordingly, no precise estimation in the strict sense. But it would play a role of a foundation stone in proposing the problem. Main purpose of the present paper is to establish for Laurent coefficients of functions from $\mathfrak{F}_{R}$ an estimation which is analogous to Littlewood's result referred to above in case of the unit circle.

Now any function $w=F(z) \in \mathfrak{F}_{R}$ maps by assumption the whole unit circumference $\mid z=1$ onto $w=1$ so that it is prolongeable analytically across $z=1$ by means of the reflection principle. The functional equation

$$
F(z) \overline{F(1 / \bar{z})} \equiv 1
$$

determining this prolongation can be expressed in terms of Laurent coefficients by the fact that an infinite matrix $\boldsymbol{C}$ with $c_{j-k}$ as $(j, k)$-element $(j$, $k=0,1,2, \cdots)$ is unitary, i. e.

$$
\boldsymbol{C} \overline{\boldsymbol{C}}^{\prime}=\boldsymbol{E},
$$

$\boldsymbol{E}$ being the infinite unit matrix, and bar and prime designating, as usual, the passage to conjugate and to transposed matrix, respectively. Thus, the family $\widetilde{F}_{k}$ can be interpreted as consisting of analytic functions $F(z)$ regular and univalent in the duplicated annulus

$$
R^{-1}<z<R,
$$

satisfying the functional equation $F(z) \overline{F(1 / \bar{z})} \equiv 1$ and the normalization $F(1)$ $=1$, and further subject to the condition that $F(z)>1$ for $z !>1$. 
In a recent paper, Nehari and Schwarz [12] have dealt with coefficient problems of univalent Laurent series. By using our notations, one of their results may be stated as follows: Let $F(z)=\sum_{n=-\infty}^{\infty} c_{n} z^{n}$ be regular and univalent in $R^{-1}<|z|<R$ (but not necessarily satisfying the condition , $F(z)=1$ for,$z \mid=1$ ) and let its all coefficients be real. Then there holds

$$
\begin{array}{r}
\left|c_{n}\right| \leqq \frac{n R^{-n}}{1-R^{-4 n}} c_{1} R\left(1+R^{-2 n-2}\right)-c_{-1} R^{-1}\left(1+R^{-2 n+2}\right) ! \\
(n= \pm 2, \pm 3, \cdots),
\end{array}
$$

and the inequality is sharp for any $n$ with $n \geqq 2$, as shown by the function

$$
\begin{aligned}
\wp\left(\lg \frac{z}{R} \mid i \pi,-2 \lg R\right) & =\sum_{n=-\infty}^{\infty} \frac{n R^{-n}}{1-R^{-4 \pi}} z^{n}+c_{0}, \\
c_{0}\left(=\frac{i \eta_{1}}{\pi}\right) & =\frac{1}{12}-2 \sum_{\nu=1}^{\infty} \frac{\nu R^{-\nu}}{1-R^{-4 \nu}},
\end{aligned}
$$

where $\Sigma^{\prime}$ designates that summation is extended over all integers $n$ save $n=0$; it is obvious that the extremal character is preserved by trivial transformations.

The extremal function maps $R^{-1}<|z|<R$ onto the whole plane cut along a half-line and a rectilinear segment both lying on the real axis. We remark here, in particular, that, if $c_{1}$ and $c_{-1}$ are supposed to be of order $O(1)$, the above inequality implies asymptotic estimations

$$
c_{n b}=O\left(n R^{-n}\right) \quad \text { as } n \rightarrow+\infty
$$

and

$$
c_{\imath l}=O\left(-n R^{n}\right) \quad \text { as } n \rightarrow-\infty,
$$

while for the extremal function mentioned above the latter estimation can be replaced by a far restrictive one, namely $c_{n}=O\left(-n R^{3 n}\right)$ as $n \rightarrow-\infty$ by virtue of a special circumstance $c_{1} R^{-1}=c_{-1} R$.

We now turn to our own discourse concerning the family $\mathfrak{F}_{R}$. The matrix equation $\boldsymbol{C} \overline{\boldsymbol{C}}^{\prime}=\boldsymbol{E}$ involves a relation $\sum_{n=-\infty}^{\infty} \mid \boldsymbol{c}_{n}^{2}=1$ implying the uniform boundedness of the coefficients. But this relation may also be obtained from the identity

$$
\sum_{n=-\infty}^{\infty} c_{n}^{2} r^{2 n}=\frac{1}{2 \pi} \int_{0}^{2 \pi} F\left(r e^{i \theta}\right)^{2} d \theta \quad\left(R^{-1}<r<R\right)
$$

by merely putting $r=1$. The last identity further implies

$$
c_{n}=o\left((R-\delta)^{-n}\right) \quad \text { as } n \rightarrow+\infty,
$$

$\delta(<R)$ being here any positive number. The same result can be deduced also from the fact that the Laurent series of $F(z) \in \mathfrak{F}_{R}$ converges itself absolutely on $z=R-\delta$. But the $o$-notation involved here is not uniform but may depend on the choice of $\delta$ even when a function $F(z)$ is fixed.

On the other hand, since there holds $F(z)<1$ for $R^{-1}<z \mid<1$, we get 


$$
\sum_{n=-\infty}^{\infty} c_{n}{ }^{2} R^{-2 n}=\frac{1}{2 \pi} \int_{0}^{2 \pi} F\left(R^{-1} e^{i \theta}\right){ }^{2} d \theta<1
$$

where $F\left(R^{-1} e^{i \theta}\right)$ denotes the radial limit $F\left((R-0)^{-1} e^{i \theta}\right)$ existent almost everywhere for $0 \leqq \theta<2 \pi$. Consequently, we can conclude, in particular, that there holds, besides an asymptotic estimation

$$
c_{n}=o\left(R^{n}\right) \quad \text { as } n \rightarrow-\infty
$$

in which the $o$-notation may depend on $F(z)$, a uniform estimation

$$
c_{n}:<R^{n} \quad \text { for } n \leqq 0 .{ }^{1)}
$$

\section{Preliminaries.}

In the final part of the preceding section we have noticed that there holds $c_{n}=0\left((R-\delta)^{-n}\right)$ as $n \rightarrow+\infty$ for any arbitrarily small positive $\delta$. But the uniformity of the $\sigma$-notation has not been ensured with respect to $\delta$. A question will arise whether we can put here $\delta=0$ or not. It is our purpose to show that, though the question is answered negatively, it is affirmatively verified after a slight modification and really in a more quantitative form. On the other hand, it will also be shown that for the coefficients with non-positive suffices, we can derive, besides an asymptotic behavior $c_{n}$ $=o\left(R^{n} / \sqrt{-n}\right)$ as $n \rightarrow-\infty$, uniform bounds more precise than those mentioned above. In fact, roughly speaking, we can conclude a pair of estimations of the form

$$
c_{n}<\left\{\begin{array}{lr}
A(R) n R^{-n} & (n=1,2, \cdots), \\
R^{n}(1-B(R)) & (n=0,-1,-2, \cdots),
\end{array}\right.
$$

where $A(R)$ and $B(R)$ are definite positive quantities depending only on $R$.

In order to see the order of preciseness of our result, it will here be noticed especially that, for $n>0, c_{n}$ can be really of order $n R^{-n}$ within the family $\widetilde{\mho}_{R}$. For that purpose we consider a function

$$
F_{R}^{*}(z)=\sum_{n=-\infty}^{\infty} c_{n}^{*} z^{n}
$$

which maps the basic annulus $1<\mid z<R$ onto the exterior of the unit circle cut along a half-line on the negative real axis terminating at the point at infinity in such a manner that $z_{i}=1$ corresponds to the whole unit circumference. Under the condition of normalization $F_{R}^{*}(1)=1$, this function $F_{R}^{*}(z)$ is uniquely determined and belongs to the family $\mathfrak{F}_{k}$.

It is further noted that the coefficients of $F_{R}^{*}(z)$ are all real. Since $F_{R}^{*}(z)$ is evidently regular throughout the closed annulus $1 \leqq z \leqq R$ except a pole of the second order lying at $z=R$, we can conclude that its Laurent coefficients with positive suffices $n$ must really be asymptotically of order $n R^{-n}$ as $n \rightarrow+\infty$.

1) We shall later derive a more precise estimation; cf theorem 8 . 
The behavior of the so-called Grötzsch's extremal function $F_{R}^{*}(z)$ will be availed especially around its pole. As shown in a previous paper [9],2) this function is expressible explicitly in terms of elliptic theta-functions:

$$
F_{R}^{*}(z)=\frac{\vartheta_{4} \vartheta_{4}(v)+\vartheta_{3} \vartheta_{3}(v)}{\vartheta_{4} \vartheta_{4}(v)-\vartheta_{3} \vartheta_{3}(v)}, \quad v=\frac{1}{2 \pi i} \lg \frac{-z}{R},
$$

theta-functions depending on the parameter $q=R^{-1}$. Transforming them into sigma-functions in the Weierstrassian theory of elliptic functions with primitive periods

$$
2 \Omega_{1}=2 \pi \quad \text { and } \quad 2 \Omega_{3}=2 i \lg R,
$$

it may be written in an alternative form

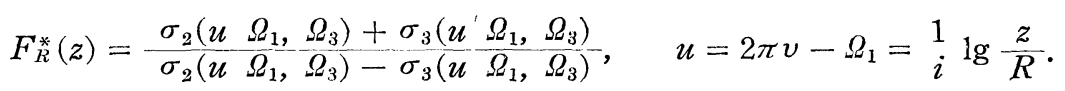

- In the following lines we shall, however, mainly use the functions concerning the primitive periods

$$
2 \omega_{1}=2 i \pi \quad \text { and } \quad 2 \omega_{3}=-4 \lg R
$$

rather than those concerning the primitive periods $2 \pi$ and $2 i \lg R$. Accordingly, the ordinary notations will be reserved for the former system while the quantities concerning the latter system will be designated, if necessary, by the capital letters corresponding to the ordinary ones._- Now the point $z=R$ corresponds to $u=0$ around which the Taylor expansions of the entire functions $\sigma_{\lambda}\left(u \Omega_{1}, \Omega_{3}\right)(\lambda=2,3)$ begin with $\sigma_{\lambda}\left(u \Omega_{1}, \Omega_{3}\right)=1-E_{\lambda} u^{2} / 2+O\left(u^{4}\right)$, $E_{\lambda}=\gamma\left(\Omega_{\lambda} \Omega_{1}, \Omega_{3}\right)$ where the $O$-notation concerns $u \rightarrow 0$. Consequently, $F_{R}^{*}(z)$ behaves in a neighborhood of $z=R$ such as

$$
F_{R}^{*}(z)=\frac{4}{E_{2}-E_{3}}-\frac{R z}{(R-z)^{2}}+O(1) ;
$$

the last $O$-notation concerns $z \rightarrow R$. Thus, the function defined by

$$
F_{R}^{*}(z)-\frac{4}{E_{2}-E_{3}} \frac{R z}{(R-z)^{2}}=\sum_{n=-\infty}^{0} c_{n}^{*} z^{n}+\sum_{n=1}^{\infty}\left(c_{n}^{*}-\frac{4}{E_{2}-E_{3}} \frac{n}{R^{n}}\right) z^{n}
$$

being regular throughout the closed annulus $R^{-1} \leqq z \leqq R$, the coefficients of the Laurent expansion of $F_{R}^{*}(z)$ must be asymptotically of the form

$$
c_{n}^{*}=\frac{4}{E_{2}-E_{3}} \frac{n}{R^{n}}+o\left(\frac{1}{R^{n}}\right) \quad \text { as } n \rightarrow \infty
$$

and, as already noticed, $c_{n}^{*}=o\left(R^{n}\right)$ as $n \rightarrow-\infty$.

Though the above analysis will suffice for our present purpose, it will be more convenient for later arguments to make use of functions concerning primitive periods

$$
2 \omega_{1}=2 i \pi \quad \text { and } \quad 2 \omega_{3}=-4 \lg R
$$

2) In [9] we have mainly considered, instead of $F_{R}^{*}(z)$ itself, the function defined by $-F_{k}^{*}(-z)$. 
as done by Nehari and Schwarz [12], rather than those with $2 \Omega_{1}=2 \pi$ and $2 \Omega_{3}=2 i \lg R_{1}$. The transference can be performed by actual calculation by means of classical formulas or more briefly in view of the uniqueness of mapping. In fact, we have readily the identity

$$
F_{R}^{*}(z)=\frac{1}{\sqrt{e_{1}-e_{3}} \sqrt{e_{2}-e_{3}}}\left(\&\left(\lg \frac{z}{R}\right)-e_{3}\right),
$$

where $e_{\lambda}(\lambda=1,2,3)$ are quantities depending, of course, on the periods $2 \omega_{1}$ and $2 \omega_{3}$, i. e., $\omega_{1}+\omega_{2}+\omega_{3}=0$ and

$$
\left.e_{\lambda}=\gamma\left(\omega_{\lambda}\right) \equiv \mathcal{\gamma}\left(\omega_{\lambda}, \omega_{1}, \omega_{3}\right) \quad(\lambda=1,2,3) .{ }^{3}\right)
$$

Consequently, the precise values of the Laurent coefficients of $F_{R}^{*}(z)$ are given by

$$
c_{n}^{*}=\frac{1}{\sqrt{e_{1}-e_{3}} \sqrt{e_{2}-e_{3}}} \frac{n R^{-n}}{1-R^{-4 n}} \quad(n= \pm 1, \pm 2, \cdots),
$$

or, based on the formulas

$$
E_{2}=-e_{3}+2 \sqrt{e_{1}-e_{3}} \sqrt{e_{2}-e_{3}} \quad \text { and } \quad E_{3}=-e_{3}-2 \sqrt{e_{1}-e_{3}} \sqrt{e_{2}-e_{3}},
$$

they can be expressed also in the from

$$
c_{n}^{*}=\frac{4}{E_{2}-E_{3}} \frac{n R^{-n}}{1-R^{-4 n}} \quad(n= \pm 1, \pm 2, \cdots),
$$

whence readily follows again the above-mentioned asymptotic expressions. As once noted, the coefficients with negative suffices behave not only such as $c_{n}^{*}=o\left(R^{n}\right)$ but really such as $c_{n}^{*}=O\left(-n R^{3 n}\right)$ as $n \rightarrow-\infty$.

Now it is a well-known fact that the function $z /(1-z)^{2}$ possesses several extremal properties within the family of functions $f(z)$ regular and univalent in the unit circle and normalized at the origin such as $f(0)=0$ and $f^{\prime}(0)=1$. In the theory of family $\mathfrak{F}_{R}$ the function $F_{R}^{*}(z)$ often plays corresponding roles. For instance, the distortion theorem of Grötzsch [8] states that there holds

$$
-F_{R}^{*}(-z) \leqq F(z) \leqq F_{R}^{*}(z) \quad(1<, z<R)
$$

for any function $F(z) \in \mathfrak{F}_{R}$ and further that the equality sign in the leftand right-hand inequalities can appear at any assigned point $z_{0}$ in 1 $<z \mid<R$ if and only if $F(z)$ is of the form

and

$$
-F_{R}^{*}\left(-z^{\prime} z_{0} / z_{0}\right) e^{-i \arg \left(-F_{R}^{*}\left(-\mid z_{0}: / z_{0}\right)\right)}
$$

$$
F_{R}^{*}\left(z, z_{0} / z_{0}\right) e^{-\imath \arg F_{R}^{*}\left(\left|z_{0}\right| / z_{0}\right)},
$$

respectively. It can be shown, on the other hand, that there exists moreover a limit-equation

$$
\lim _{R \rightarrow \infty} \frac{F_{R}^{*}(z)}{R}=\frac{z}{(1-z)^{2}}
$$

3) There holds $e_{1}<e_{2}<e_{3}$ while $E_{1}>E_{2}>E_{3}$. 
valid for $0<\mid z:<1$ uniformly in the wider sense; cf. [9].

In spite of such being the circumstance, the function $F_{R}^{*}(z)$ does not play a role of extremal function at least for some suffices $n$ in our coefficient problem on $\widetilde{\mho}_{R}$, contrary to Bieberbach's conjecture on functions univalent in the unit circle. For instance, it is evident that the best possible estimation for $c_{1}$ is given by

$$
{ }^{\prime} c_{1} \leqq 1
$$

with a unique extremal function $z$ which is not identical with $F_{R}^{*}(z)$. Moreover, since the identity $\sum_{n=-\infty}^{\infty} c_{n}{ }^{2}=1$ remains always to hold, any function $F(z) \in \widetilde{F}_{R}$ cannot be a universal extremal function for our coefficient problem. However, it appears perhaps to be reasonable to propose a conjecture that Laurent coefficients of any function belonging to $\mathfrak{F}_{R}$ would be asymptotically majorized by $c_{n}^{*}$ at least for sufficiently large $n$.

\section{Main results.}

We shall now proceed to our main disccourse. Our tools of attacking the problem are quite elementary. In fact, from the integral representation of Laurent coefficients

$$
c_{n}=\frac{1}{2 \pi i} \int_{|z|=r} \frac{F(z)}{z^{n+1}} d z \quad\left(R^{-1}<r<R ; n=0, \pm 1, \pm 2, \cdots\right)
$$

we have readily a system of inequalities of Cauchy type

$$
c_{n} \leqq \frac{1}{r^{n}} \frac{1}{2 \pi} \int_{0}^{2 \pi} F\left(r e^{i \theta}\right) d \theta \quad(n=0, \pm 1, \pm 2, \cdots)
$$

valid for any $r$ with $R^{-1}<r<R$. Consequently, for any fixed $n$, whenever once an upper bound $C_{n}(r)$ of the right-hand member of the last inequality is known for some $r$, there results immediately a corresponding estimation

$$
c_{n} \leqq C_{n}(r) \text {. }
$$

Especially, if $C_{n}(r)$ is obtained for any $r$ belonging to a sub-interval $J$ of $R^{-1}<r<R$, then it can be concluded that there holds

$$
c_{n} \leqq \inf _{r \in J} C_{n}(r) \text {. }
$$

According to the just explained course, in order to estimate the integral of $F\left(r e^{2 \theta}\right)$ over $0 \leqq \theta<2 \pi$, we shall here make use of a theorem of Prawitz [13] which can be stated as follows:

Suppose a ring domain laid on the $w=\rho e^{i \varphi}$-plane be bounded by two analytic contours $\Gamma_{1}$ and $\Gamma_{2}$ such that $\Gamma_{1}$ lies in the finite simply-connected domain bounded by $\Gamma_{2}$ alone and that $\Gamma_{1}$ itself (and hence $\Gamma_{2}$ also) surrounds the origin. Let further $g(\rho)$ be a function taking non-negative values alone and increasing with $\rho$. Then there holds an inequality

$$
\int_{\Gamma_{1}} g(\rho) d \varphi \leqq \int_{\Gamma_{2}} g(\rho) d \varphi,
$$


the integrals being to be taken in the positive sense with respect to the interiors of respective contours.

It is to be noted further that, as shows the own proof given by Prawitz, we may permit in the assumption of the theorem the limiting case where $\Gamma_{1}$ touches $\Gamma_{2}$ at some points.

In the following lines, based on our special normalization that any function $w=F(z) \in \mathfrak{F}_{R}$ maps $z=1$ onto $w=1$, the upper bounds of $c_{n}$ to be obtained for positive and non-positive suffices will be of somewhat different nature. Accordingly, distinguishing these two systems, we shall state below our main result separately.

THEOREM 1. For any $F(z) \in \mathfrak{\mho}_{R}$ there holds an estimation

$$
c_{n} \leqq \operatorname{Min}_{1 \leq r<R} C_{n}(r) \quad(n=1,2, \cdots)
$$

where $C_{n}(r)$ is defined by

$$
C_{n}(r)=\frac{1}{r^{n}}\left\{\frac{1}{\sqrt{ } e_{1}-e_{3} \sqrt{e_{2}-e_{3}}}\left(\zeta\left(\lg \frac{R}{r}\right)-e_{3} \lg r-\zeta(\lg R)\right)+1\right\} .
$$

Proof. Remembering the distortion theorem of Grötzsch, we may put in the theorem of Prawitz re-stated above $g(\rho) \equiv \rho$ and

$$
\begin{aligned}
& \Gamma_{1} \text { : } \\
& \rho e^{i \varphi}=F\left(r e^{i \theta}\right) \\
& (0 \leqq \theta<2 \pi), \\
& \Gamma_{2} \text { : } \\
& \rho e^{i \varphi}=F_{R}^{*}(r) e^{i \theta} \\
& (0 \leqq \theta<2 \pi) \text {. }
\end{aligned}
$$

We thus obtain an inequality

$$
\frac{1}{2 \pi} \int_{0}^{2 \pi} F\left(r e^{i \theta}\right) d \arg F\left(r e^{i \theta}\right) \leqq F_{R}^{*}(r)
$$

valid for any $r$ with $1 \leqq r<R$. By virtue of Cauchy-Riemann equation, we have

$$
d \arg F\left(r e^{i \theta}\right)=\frac{\partial \arg F\left(r e^{i \theta}\right)}{\partial \theta} d \theta=\frac{\partial \lg F\left(r e^{i \theta}\right)}{\partial \lg r} d \theta
$$

so that the last inequality becomes

$$
r \frac{d}{d r} \frac{1}{2 \pi} \int_{0}^{2 \pi} F\left(r e^{i \theta}\right) d \theta \leqq F_{K}^{*}(r) .
$$

Integration with respect to $r$, after divided by $r$, leads in view of $\left|F\left(e^{i \theta}\right)\right|=1$ readily to

$$
\begin{aligned}
\frac{1}{2 \pi} \int_{0}^{3 \pi} F\left(r e^{2 \theta}\right) d \theta-1 & \leqq \int_{1}^{r} F_{R}^{*}(r) \frac{d r}{r}=\int_{1}^{r} \frac{-\gamma\left(\lg \frac{R}{r}\right)+e_{3}}{\sqrt{e_{1}-e_{3}} \sqrt{e_{2}-e_{3}}} d \lg \frac{R}{r} \\
& =\frac{1}{\sqrt{e_{1}-e_{3}} \sqrt{e_{2}-e_{3}}}\left(\zeta\left(\lg \frac{R}{r}\right)-e_{3} \lg r-\zeta(\lg R)\right)
\end{aligned}
$$

and hence 


$$
c_{n} \leqq \frac{1}{r^{n}} \frac{1}{2 \pi} \int_{0}^{2 \pi} F\left(r e^{2 \theta}\right), d \theta \leqq C_{n}(r) \quad(1 \leqq r<R ; n=1,2, \cdots) .
$$

It is evident that, for any fixed $n>0, C_{n}(r)$ is a function of $r$ continuous for $1 \leqq r<R$ and satisfying the limit-equation $C_{n}(R-0)=\infty$. Hence, the infimum of $C_{n}(r)$ for $1 \leqq r<R$ is really attained at some value of $r$ in $1 \leqq r<R$. The theorem has been thus proved.

The bound in the theorem just established is expressed in terms of transcendental quantities. It will be of some interest to derive its asymptotic behavior as $n$ increases.

THEOREM 2. For any $F(z) \in \mathfrak{F}_{R}$ there holds an asymptotic estimation

$$
c_{n}<\frac{e}{\sqrt{e_{1}-e_{3}} \sqrt{e_{2}-e_{3}}} \frac{n}{R^{n}}+O\left(\frac{1}{R^{n}}\right)
$$

valid for $n \rightarrow+\infty$, the $O$-notation being uniform with respect to $n$ as well as $F(z)$. By the way, we notice here an identity

$$
\sqrt{\sqrt{e_{1}-e_{3}} \sqrt{e_{2}-e_{3}}}=R \prod_{\nu=1}^{\infty}\left(1-\frac{1}{R^{4 \nu}}\right)^{-4}\left(1+\frac{1}{R^{2 \nu}}\right)^{-4}=R\left(\sum_{\nu=1}^{\infty} \frac{1}{R^{\nu(\nu-1)}}\right)^{-4} .
$$

Proof. From the expression for $C_{n}(r)$ given in theorem 1 it follows readily that there holds an asymptotic relation

$$
C_{n}(r)=\frac{1}{r^{n}}\left(\frac{1}{\sqrt{e_{1}-e_{3} \sqrt{e_{2}-e_{3}}}} \frac{R}{\lg \frac{R}{r}}+O(1)\right)
$$

as $r \rightarrow R-0$, the $O$-notation depending, of course, on the particular function $F_{R}^{*}(z)$ alone. On the other hand, if we put $r=R e^{-1 / n}$, then $r$ tends to $R-0$ as $n \rightarrow \infty$. Hence, substituting this value of $r$, we get

$$
C_{n}\left(R e^{-1 / 3}\right)=\frac{e}{R^{n}}\left(\frac{n}{\sqrt{e_{1}-e_{3}} \sqrt{e_{2}-e_{3}}}+O(1)\right),
$$

whence follows the desired estimation.

In the proof of theorem 1 Prawitz's inequality has played an essential role. As an alternative inequality we could have taken into account the Cauchy's, i. e.,

$$
c_{n} \leqq \frac{1}{r^{n}} F_{R}^{*}(r)
$$

However, this inequality would imply an asymptotic relation

$$
\mid c_{n} \leqq \frac{1}{r^{n}}\left(\frac{1}{\sqrt{e_{1}-e_{3}} \sqrt{e_{2}-e_{3}}} \frac{1}{\left(\lg \frac{\bar{R}}{r}\right)^{2}}+O(1)\right)
$$

as $r \rightarrow R-0$, whence follows, by minimizing the right-hand member regarded as a function of $r$ for $1<r<R$, namely by putting $r=R e^{-2 / n}+o(1)$, merely an estimation 


$$
c_{n} \leqq \frac{e^{2} / 4}{\sqrt{e_{1}-e_{3}} \sqrt{e_{2}-e_{3}}} \frac{n^{2}}{R^{n}}+O\left(\frac{1}{R^{n}}\right)
$$

which is far weaker than that given in theorem 2 .

REMARK. Estimations stated in theorems 1 and 2 remain to hold also for Laurent coefficients of any function regular and univalent in $1<z<R$, which maps $z=R$ onto a continuum exterior to the unit circumference and $|z|=1$ onto a continuum belonging to the closed unit disc. In fact, any such function $F(z)$ satisfies the distortion inequality $F(z) \leqq F_{R}^{*}(z)$, as readily shown by means of Schwarz lemma, and hence the proofs of these theorems remain valid without any alteration.

We next proceed to deal with the problem concerning the coefficients with non-positive suffices.

THEOREM 3. For any $F(z) \in \mathfrak{F}_{R}$ there holds an inequality

$$
\sum_{n=-\infty}^{\infty} c_{n}{ }^{2} R^{-2 n} \leqq \frac{e_{2}-e_{3}}{e_{1}-e_{3}}<1,
$$

whence follows especially an asymptotic estimation $c_{n}=o\left(R^{n}\right)$ as $n \rightarrow-\infty$ in which the o-notation may depend on $F(z)$ and further follows a uniform estimation

$$
\left.c_{n} \mid \leqq R^{n} \frac{-\sqrt{e_{2}-e_{3}}}{\sqrt{e_{1}-e_{3}}}<\operatorname{Min}\left\{4 R^{n-1}, R^{n}\right\} \quad(n=0,-1,2-, \cdots) .^{4}\right)
$$

Here we notice an identity

$$
\begin{aligned}
\frac{-\sqrt{e_{2}-e_{3}}}{\sqrt{e_{1}-e_{3}}} & =\frac{4}{R} \prod_{\nu=1}^{\infty}\left(\frac{1+R^{-4 \nu}}{1+R^{-4 \nu+2}}\right)^{4} \\
& =\frac{1}{R}\left(1+2 \sum_{\nu=1}^{\infty} \frac{1}{R^{2 \nu(\nu+1)}}\right)^{2}\left(1+2 \sum_{\nu=1}^{\infty} \frac{1}{R^{2 \nu^{2}}}\right)^{-2} .
\end{aligned}
$$

Proof. Let $R^{-1} \leqq r \leqq 1$ and $z=r$. Then, in view of the functional equation $F(z) F(1 / \bar{z})=1$, together with a distortion inequality, we get

$$
F(z)=\frac{1}{F(1 / \bar{z})} \leqq \frac{1}{-F_{R}^{*}(-1 / r)}=-F_{R}^{*}(-r)
$$

and consequently we conclude

$$
\sum_{n=-\infty}^{\infty} c_{n}{ }^{2} R^{-2 n}=\frac{1}{2 \pi} \int_{0}^{2 \pi} F\left(R^{-1} e^{2 \theta}\right)^{2} d \theta \leqq\left(-F_{R}^{*}\left(-R^{-1}\right)\right)^{2}<1 .
$$

But we have

$$
\begin{aligned}
-F_{R}^{*}\left(-R^{-1}\right) & =-\frac{\wp\left(\lg \left(-R^{2}\right)\right)-e_{3}}{\sqrt{e_{1}-e_{2} \sqrt{e_{2}}-e_{3}}}=\frac{-\sqrt{e_{2}-e_{3}}}{\sqrt{e_{1}-e_{3}}} \\
& =\frac{4}{R} \prod_{\nu=1}^{\infty}\left(\frac{1+R^{-4 \nu}}{1+R^{-4 \nu+2}}\right)^{4}<\frac{4}{R},
\end{aligned}
$$

4) Cf. theorem 8 below. 
which proves the theorem.

REMARK. In a paper of Teichmüller [15], we find that the behavior of the ratio $\Phi(\boldsymbol{P}) / \boldsymbol{P}$ plays an important role, where $\lg \boldsymbol{\Phi}(\boldsymbol{P})$ denotes the modulus of a Grötzsch's extremal domain consisting of the exterior of the unit circle cut along a half-line on the positive real axis which starts at a point $\boldsymbol{P}(>1)$. In a previous paper [9], we have concerned this problem giving an alternative proof of the fact that there hold the following relations:

$$
\Phi(\boldsymbol{P})<4 \boldsymbol{P} \quad \text { and } \quad \lim _{P \rightarrow \infty} \frac{\Phi(\boldsymbol{P})}{\boldsymbol{P}}=4 .
$$

The proof was based on a parametric representation

$$
\Phi(\boldsymbol{P})=R, \quad \boldsymbol{P}=-F_{R}^{*}(-R)=\frac{1+k^{\prime}}{1-k^{\prime}},
$$

where $k^{\prime}$ denotes the complementary modulus of sn-function with primitive periods $2 \pi$ and $2 i \lg R$. But, as shown in the above proof of theorem 3 , if the use is made of the quantities concerning the primitive periods $2 i \pi$ and $-4 \lg R$, we can verify this fact more straightforward. In fact, we then have

$$
\begin{gathered}
\Phi(\boldsymbol{P})=R, \quad \boldsymbol{P}=-F_{R}^{*}(-R)=\frac{1}{-F_{R}^{*}\left(-R^{-1}\right)}=\frac{R}{4} \prod_{\nu=1}^{\infty}\left(\frac{1+R^{-4 \nu+2}}{1+R^{-4 \nu}}\right)^{4} ; \\
\frac{\Phi(\boldsymbol{P})}{\boldsymbol{P}}=4 \prod_{\nu=1}^{\infty}\left(\frac{1+R^{-4 \nu}}{1+R^{-4 \nu+2}}\right)^{4}=R \frac{-\sqrt{e_{2}-e_{3}}}{\sqrt{e_{1}-e_{3}}} .
\end{gathered}
$$

However, it is also readily verified that two expressions availed above for $\boldsymbol{P}$ are really identical. In fact, based on the relations $\omega_{1}=i \Omega_{1}$ and $\omega_{3}=i \Omega_{3}$, we have

$$
\frac{1+k^{\prime}}{1-k^{\prime}}=\frac{\sqrt{E_{1}-E_{3}}+\sqrt{E_{2}-E_{3}}}{\sqrt{E_{1}-E_{3}}-\sqrt{E_{2}-E_{3}}}=\frac{\sqrt{e_{1}-e_{3}}}{-\sqrt{e_{2}-e_{3}}} .
$$

It would be noticed that the estimation $c_{n} \leqq R^{n}\left(-F_{R}^{*}\left(-R^{-1}\right)\right)$ contained in theorem 3 is also a consequence of Cauchy's inequality. Indeed, we readily get, for any $r$ with $R^{-1}<r \leqq 1$, an inequality

$$
c_{n i}{ }^{\prime} \leqq \frac{1}{r^{n}} \operatorname{Max}_{0 \leq \theta<2 \pi} F\left(r e^{\imath \theta}\right) \leqq \frac{1}{r^{n}}\left(-F_{R}^{*}(-r)\right)
$$

which implies, for $r \rightarrow R^{-1}$, the desired estimation. As mentioned in a remark subsequent to theorem 2, if we use Cauchy's inequality instead of Prawitz's, there follows only a far weaker estimation for $c_{n}$ with a positive suffix, while, contrary to this and as actually shown above, Cauchy's inequality has been availed with some effect for estimating $c_{n}$ with a non-positive suffix. But Prawitz's inequality may also be used for obtaining an alternative form of estimation in this case. The result will be formulated as in the following theorem. 
THEOREM 4. For any $F(z) \in \mathfrak{F}_{R}$ there holds

$$
\begin{aligned}
& c_{n} \leqq R^{n}\left\{1-\sqrt{\sqrt{e_{1}-e_{3}} \sqrt{e_{2}}-e_{3}}\left(\zeta(\lg R)+\eta_{3}-e_{3} \lg R\right)\right\} \\
&=R^{n}\left\{1-\frac{1}{\sqrt{e_{1}-e_{3}} \sqrt{e_{2}}-e_{3}}\left(\frac{1}{R-1}-\sum_{\nu=1}^{\infty} \frac{R^{-3 v}}{1+R^{-2 \nu}}-e_{3} \lg R\right)\right\} \\
&(n=0,-1,-2, \cdots) .
\end{aligned}
$$

Proof. Let $R^{-1}<r \leqq 1$ and $z=r$. Then, similarly as in the proof of the preceding theorem, we get

$$
F(z)=\underset{F(1 / \bar{z}),}{1} \geqq \frac{1}{F_{R}^{*}(1 / r)}=F_{R}^{*}(r) .
$$

Consequently, by applying Prawitz's theorem in a similar manner as in the proof of theorem 1, we obtain

$$
r \frac{d}{d r} \frac{1}{2 \pi} \int_{0}^{2 \pi} F\left(r e^{2 \theta}\right) d \theta=\frac{1}{2 \pi} \int_{0}^{2 \pi} F\left(r e^{i \theta}\right) d \arg F\left(r e^{i \theta}\right) \geqq F_{R}^{*}(r),
$$

whence readily follows

$$
1-\frac{1}{2 \pi} \int_{0}^{2 \pi} F\left(r e^{2 \theta}\right) d \theta \geqq \int_{r}^{1} F_{R}^{*}(r) \frac{d r}{r}
$$

and hence

$$
c_{n} \leqq \frac{1}{r^{n}}\left(1-\int_{r}^{1} F_{R}^{*}(r) \frac{d r}{r}\right) \quad\left(R^{-1}<r \leqq 1 ; n=0,-1,-2, \cdots\right) .
$$

The last estimate is, for any fixed $n \leqq 0$, a continuous function of $r$ for $R^{-1} \leqq r \leqq 1$ and its minimum in this interval is evidently attained at $r=R^{-1}$. Thus we get

$$
\begin{aligned}
& c_{\eta \imath} \leqq R^{n}\left(1-\int_{1 / R}^{1} F_{R}^{*}(r) \frac{d r}{r}\right) \\
&=R^{n}\left\{1-\frac{1}{\sqrt{e_{1}-e_{3}} \sqrt{ }} \sqrt{e_{2}-e_{3}}\left(\zeta(\lg R)+\eta_{3}-e_{3} \lg R\right)\right\} \\
& \quad(n=0,-1,-2, \cdots) .
\end{aligned}
$$

REMARK. We can consider a sub-family of $\mathfrak{F}_{R}$, designated by $\mathfrak{F}_{R}(M)$, which consists of all functions $F(z) \in \widetilde{\mho}_{R}$ subject to an accessory condition of boundedness expressed by

$$
F(z)<M \quad \text { for } \quad 1<z,<R
$$

It is evidently to be supposed that there holds $M \geqq R$. For any function $F(z) \in \mathfrak{F}_{R}(M)$, we have the distortion inequalities

$$
-F_{R}^{*}(-z ; M) \leqq F(z) \leqq F_{R}^{*}(z ; M)
$$

where the extremal function $F_{R}^{*}(z ; M) \in \mathfrak{F}_{R}(M)$ is defined by 


$$
F_{R}^{*}(z ; M)=F_{M}^{*-1}\left(F_{R}^{*}(z)\right),
$$

$F_{M}^{*-1}$ denoting the function inverse to $F_{M}^{*}$. The function $w=F_{R}^{*}(z ; M)$ maps the basic annulus $1<z<R$ onto the annulus $1<w<M$ cut along the rectilinear slit connecting two points $-M$ and $F_{M}^{*-1}\left(F_{R}^{*}(-R)\right)$.

Since the distortion inequalities are known, the argument employed in the proof of theorem 1 leads to a corresponding result on Laurent coefficients of functions from $\mathfrak{F}_{R}(M)$. Namely, we can conclude that for any $F(z)$ $\in \mathfrak{F}_{R}(M)$ there holds an estimation

$$
c_{n} \leqq \operatorname{Min}_{1 \leq r \leq R} \frac{1}{r^{n}}\left(\int_{1}^{r} F_{R}^{*}(r ; M) \frac{d r}{r}+1\right) \quad(n=1,2, \cdots) .
$$

We get, in particular,

$$
c_{n} \leqq \operatorname{Min}\left\{1, \frac{1}{R^{n}}(M \lg R+1)\right\} \quad(n=1,2, \cdots) .
$$

Analogues of theorems 3 and 4 can also be obtained similarly.

\section{Elementary estimates.}

The estimate given in theorem 1 has been expressed in terms of transcendental quantities. On the other hand, while the asymptotic estimate given in theorem 2 is of elementary nature, it is still accompanied by a remainder term, though this remainder can be expressed explicitly in the form of infinite series with elementary terms. We shall now attempt to derive an alternative estimate for $c_{n}$ with $n>0$, which is slightly rough but of completely elementary nature and closed in form.

We first consider a rational function defined by

$$
\lambda(z)=\frac{\left(R^{2}-1\right)^{2}}{R^{2}+1} \quad(R-z)^{2}-\frac{2 R}{R^{2}+1} .
$$

The annulus $1<z<R$ is mapped by $Z=\lambda(z)$ univalently onto a doublyconnected domain symmetric with respect to the real axis whose boundary consists of a half-line extending from $\lambda(-R)$ to $\infty$ along the negative real axis and a closed curve $\Gamma$ surrounding the origin; further $z=1$ corresponds to $Z=1$. It is seen that the contour $\Gamma$ contains in its interior the unit circle. In fact, we have

$$
\begin{aligned}
\lambda\left(e^{i \theta}\right) & =\left|\frac{\left(R^{2}-1\right)^{2}}{R^{2}+1} \frac{e^{2 \theta}}{\left(R-e^{i \theta}\right)^{2}}-\frac{2 R}{R^{2}+1}\right| \\
& =\left(1+\frac{4 R^{2}\left(R^{2}-1\right)^{2} \sin ^{2} \theta}{\left(R^{2}+1\right)^{2}\left(R^{2}-2 R \cos \theta+1\right)^{2}}\right)^{1 / 2} \geqq 1 .
\end{aligned}
$$

Based on the symmetry character of $\Gamma$, it is possible to map the simplyconnected domain consisting of the exterior of $\Gamma$ onto the exterior of the unit circle in such a mannar that the parts lying in the upper half-planes correspond each other and the three points \pm 1 and $\infty$ remain invariant. The last-mentioned conditions determine the mapping function uniquely 
which will be designated by $\kappa(Z)$.

Since the whole unit disc $Z<1$ lies in the interior of $\Gamma$, the principle of maximum modulus, or rather briefly the Schwarz's lemma applied to the inverse function $\kappa^{-1}(w)$ with respect to $w>1$, implies that the inequality

$$
\kappa(Z)^{\prime}<Z
$$

remains to hold provided $1<|\kappa(Z)|<\infty$.

But, by virtue of the unicity of mapping, we have the identity

$$
F_{R}^{*}(z) \equiv \kappa(\lambda(z))
$$

whence follows a comparison inequality

$$
F_{R}^{*}(z)<\lambda(z) \quad \text { for } 1<z<R .
$$

Thus, a majorant of $F_{R}^{*}(z)$ having been obtained, we can substitute it into the bound given in theorem 1 , yielding the following theorem.

THEOREM 5. For any $F(z) \in \mathfrak{F}_{R}$ there holds

$$
c_{n} \mid \leqq \operatorname{Min}_{1 \leq r<R} \frac{\Lambda(r)}{r^{n}}
$$

where $\Lambda(r)$ is defined by

$$
\begin{array}{r}
\Lambda(r)=\int_{1}^{r} \lambda(r) \frac{d r}{r}+1=\frac{\left(R^{2}-1\right)^{2}}{R^{2}+1}\left(\frac{1}{R-r}-\frac{1}{R-1}\right) \\
-\frac{2 R}{R^{2}+1}(r-1)+1 ;
\end{array}
$$

in particular,

$$
\begin{aligned}
c_{n} & \leqq-\frac{e}{R^{n}} \Lambda\left(R e^{-1 / n}\right) \\
& <\frac{e}{R^{n-1}}\left(\frac{\left(R^{2}-1\right)^{2}}{R^{2}\left(R^{2}+1\right)} n-\frac{2 R^{3}-R^{2}-2 R-1}{R^{2}\left(R^{2}+1\right)}+\frac{2 R}{R^{2}+1} \frac{1}{n}\right)
\end{aligned}
$$

provided $n>1 / \lg R$.

Proof. The inequality $F_{R}^{*}(r)<\lambda(r)$ for $1<r<R$ obtained just above leads, in view of theorem 1 , readily to an estimation

$$
c_{n \imath} \leqq \frac{1}{r^{n}}\left(\int_{1}^{r} \lambda(r) \frac{d r}{r}+1\right)=\frac{\Lambda(r)}{r^{n}}
$$

valid for any $r$ with $1 \leqq r<R$. Putting especially $r=R e^{-1 / n}$ in the last relation provided $n>1 / \lg R$ and then remembering elementary inequalities

$$
\frac{1}{1-e^{-1 / n}}<n+1 \quad \text { and } \quad-e^{-1 / n}<-\left(1-\frac{1}{n}\right)
$$

valid for any $n>0$, we get 


$$
\begin{aligned}
c_{n} i & \leqq \frac{e}{R^{n}} \Lambda\left(R e^{-1 / n}\right) \\
& <\frac{e}{R^{n}}\left(\frac{\left(R^{2}-1\right)^{2}}{R^{2}+1}\left(\frac{n+1}{R}-\frac{1}{R-1}\right)-\frac{2 R}{R^{2}+1}\left(R\left(1-\frac{1}{n}\right)-1\right)+1\right) \\
& =\frac{e}{R^{n-1}}\left(\frac{\left(R^{2}-1\right)^{n}}{R^{2}\left(R^{2}+1\right)} n-\frac{2 R^{3}-R^{2}-2 R-1}{R^{2}\left(R^{2}+1\right)}+\frac{2 R}{R^{2}+1} \frac{1}{n}\right) .
\end{aligned}
$$

By the way, it may be noted that a similar method can be used also for obtaining an elementary estimate of $c_{n}$ with a non-positive suffix. In fact, for $R^{-1}<r<1$, we have an inequality

$$
F_{R}^{*}(r)=\frac{1}{F_{R}^{*}(1 / r)}>\frac{1}{\lambda(1 / r)},
$$

whence follows, based on theorem 4 , an estimation

$$
c_{n} \mid<R^{n}\left(1-\int_{R^{-1}}^{1} \frac{1}{\lambda(1 / r)}-\frac{d r}{r}\right)
$$

or, by actually evaluating the last integral,

$$
\begin{aligned}
c_{n}:< & R^{n-1}\left(R^{2}+1\right) \\
& \times\left(\frac{R^{2}-1}{\sqrt{\left(R^{2}+1\right)^{2}+4 R^{2}}} \lg \frac{(R+1)^{2}+\sqrt{\left(R^{2}+1\right)^{2}+4 R^{2}}}{2 \sqrt{R\left(R^{2}+1\right)}}-\frac{1}{2} \lg R\right) \\
& \quad(n \leqq 0) .
\end{aligned}
$$

However, the last estimate is, compared with that stated in theorem 3, not so precise. For instance, for $R \rightarrow \infty$ the estimate just derived behaves such as $R^{n-1}\left(2 \lg R-1+O\left(R^{-1}\right)\right)$.

On the other hand, we could have availed, instead of $\lambda(z)$, another analogous majorant, for instance, defined by

$$
\mu(z)=\frac{(R+1)^{2} z}{(R-z)^{2}} .
$$

But this would lead only to more rough estimations

$$
\begin{aligned}
c_{n} & \leqq \operatorname{Min}_{1 \leq r<R} \frac{1}{r^{n}}\left((R+1)^{2}\left(\frac{1}{R-r}-\frac{1}{R-1}\right)+1\right) \\
& <\frac{e}{R^{n}}\left(\frac{(R+1)^{2}}{R} n-\frac{3 R+1}{R(R-1)}\right) \quad \text { for } n>0
\end{aligned}
$$

and

$$
c_{n}<R^{n^{3}} \frac{2}{(R+1)^{2}}(R \lg R+R+1) \quad \text { for } n \leqq 0 .
$$

\section{Consequences from area-principle.}

For analytic functions univalent and suitably normalized in the interior or the exterior of a circle, a classical way to develop a unified theory is based 
on the so-called area-principle given first by Gronwall [7] and rediscovered soon later by Bieberbach [2], Faber [5] and others. For the family $\mathfrak{F}_{R}$ under consideration we can derive an analogous area-relation in terms of Laurent coefficients which will be availed with some effect for our coefficient problem.

THEOREM 6. Let

$$
F(z)=\sum_{n=-\infty}^{\infty} c_{n} z^{n} \in \mathfrak{\mho}_{R}
$$

and $A(r) \equiv A(r ; F)$ denote the area of the finite region laid on the $w$-plane and enclosed by the contour $w=F\left(r e^{i \theta}\right)(0 \leqq \theta<2 \pi)$, then there holds an identity

$$
A(r)=\pi \sum_{n=-\infty}^{\infty} n^{\prime} c_{n}^{2} r^{2 n} \quad\left(R^{-1}<r<R\right),
$$

which remains still true for $r=R^{-1}$ and $r=R$ if interpreted such as $A\left(R^{-1}\right)$ $=A\left((R+0)^{-1}\right)(\geqq 0)$ and $A(R)=A(R-0)(\leqq \infty)$, respectively.

Proof. We get in turn, for any $r$ with $R^{-1}<r<R$,

$$
\begin{aligned}
A(r) & =\int_{0}^{2 \pi} \frac{1}{2}\left(F\left(r e^{i \theta}\right)+\overline{F\left(r e^{i \theta}\right)}\right) \frac{1}{2 i} \frac{\partial}{\partial \theta}\left(F\left(r e^{i \theta}\right)-F\left(r e^{i \theta}\right)\right) d \theta \\
& =\int_{0}^{2 \pi} 12 \sum_{\mu=-\infty}^{\infty}\left(c_{\mu} e^{i \mu \theta}+\bar{c}_{\mu} e^{-i \mu}\right) \frac{1}{2 i} \sum_{\nu=-\infty}^{\infty} \nu r^{\nu}\left(c_{\nu} e^{i \nu \theta}+c_{\nu} e^{-i \nu \theta}\right) d \theta \\
& =\pi \sum_{n=-\infty}^{\infty} n c_{n}{ }^{2} r^{2 n},
\end{aligned}
$$

here multiplication (of Cauchy type) of infinite series and termwise integration of the resulting series being justified since the series converge for fixed $r$ absolutely and uniformly with respect to $\theta$. Finally, the expression representing $A(r)$ is the difference of two series with positive (more precisely, non-negative) terms alone, of which one (corresponding to negative suffices) converges for $R^{-1}<r<\infty$ while another (corresponding to positive suffices) converges for $0 \leqq r<R$. From this fact it is verified that the passage to limits $r \rightarrow(R \pm 0)^{\mp 1}$ is admitted as stated in the theorem.

It would be noted that the expression for $A(r)$ just derived is, contrary to Gronwall-Bieberbach's, not a single series with terms of the same sign, but is the difference of series of such nature. Accordingly, it will be not immediate to separate an estimation for a single coefficient. But there follows readily as a corollary of theorem 6 the following theorem which involves a noteworthy fact that the coefficients with positive suffices dominate as the whole against those with negative suffices.

THEOREM 7. The Laurent coefficients of $F(z) \in \mathfrak{F}_{R}$ satisfies inequalities

$$
\begin{array}{ll}
\left(-F_{R}^{*}(-r)\right)^{2}<\sum_{n=1}^{\infty} n c_{n}{ }^{2} r^{2 n}-\sum_{n=1}^{\infty} n c_{-n}{ }^{2} r^{-2 n}<F_{R}^{*}(r)^{2} & (1<r<R), \\
F_{R}^{*}(r)^{2}<\sum_{n=1}^{\infty} n^{*} c_{n}{ }^{2} r^{2 n}-\sum_{n=1}^{\infty} n c_{-n}{ }^{2} r^{-2 n}<\left(-F_{R}^{*}(-r)\right)^{2} & \left(R^{-1}<r<1\right)
\end{array}
$$


and an equality

$$
\sum_{n=1}^{\infty} n c_{n}^{2}-\sum_{n=1}^{\infty} n c_{-n}^{2}=1
$$

Proof. In view of Grötzsch's distortion theorem, we have

$$
\begin{array}{lc}
\pi\left(-F_{k}^{*}(-r)\right)^{2}<A(r)<\pi F_{R}^{*}(r)^{2} & (1<r<R), \\
\pi F_{R}^{*}(r)^{2}=\pi \frac{1}{F_{R}^{*}(1 / r)^{2}}<A(r) & \\
<\pi \frac{1}{\left(-F_{R}^{*}(-1 / r)\right)^{2}}=\pi\left(-F_{R}^{*}(-r)\right)^{2} \\
\quad\left(R^{-1}<r<1\right),
\end{array}
$$

vielding the esired inequalities. That the inequality sign holds here everywhere in the strict sense is justified by the fact that the image-curve of Grötzsch's extremal function corresponding to a circle $z=r$ with any $r \neq 1$ does not coincide with a circle. On the other hand, the last equality of the theorem is a consequence of normalization $F(z)=1$ along $z=1$ implying $A(1)=\pi$.

By combining the result just obtained with previously established ones, we can derive certain consequences on individual coefficients. For instance, by taking theorem 1 into account, the content of theorem 3 can be replaced partly by more precise estimations, as shown in the following theorem.

THEOREM 8. For any $F(z) \in \mathfrak{\mho}_{R}$ there holds an inequality

$$
\sum_{n=1}^{\infty} n c_{-n}{ }^{2} R^{2 n} \leqq \operatorname{Min}_{1 \leq r<R} \frac{R^{2} r^{2}}{\left(R^{2} r^{2}-1\right)^{2}}\left(\int_{1}^{r} F_{R}^{*}(r) \frac{d r}{r}+1\right)^{2} .
$$

In particular, we get

and hence

$$
\sum_{n=1}^{\infty} n\left|c_{-n}\right|^{2} R^{2 n} \leqq \frac{R^{2}}{\left(R^{2}-1\right)^{2}}
$$

$$
\begin{array}{lr}
\left|c_{n}\right| \leqq \frac{R^{n-1}}{\sqrt{-n\left(R^{2}-1\right)}} & (n=-1,-2, \cdots), \\
c_{n}=o\left(\frac{R^{n}}{\sqrt{-n}}\right) & \text { as } n \rightarrow-\infty .
\end{array}
$$

Proof. The second inequality of theorem 7 implies, by letting $r \rightarrow(R+0)^{-1}$ (cf. the final remark in theorem 6), the inequality

$$
0=F_{R}^{*}\left(-R^{-1}\right)^{2} \leqq \sum_{n=1}^{\infty} n\left|c_{n}\right|^{2} R^{-2 n}-\sum_{n=1}^{\infty} n\left|c_{-n}\right|^{2} R^{2 n} .
$$

On the other hand, we know by theorem 5 that the inequality holds good for any $r$ with $1 \leqq r<R$. Hence, for any such $r$, we get the estimation

$$
\begin{aligned}
& \sum_{n=1}^{\infty} n\left|c_{-n}\right|^{2} R^{2 n} \leqq \sum_{n=1}^{\infty} n ! c_{n}{ }^{2} R^{-2 n} \\
& \quad \leqq \sum_{n=1}^{\infty} n R^{-2 n} r^{-2 n}\left(\int_{1}^{r} F_{R}^{*}(r) \frac{d r}{r}+1\right)^{2}=\frac{R^{2} r^{2}}{\left(R^{2} r^{2}-1\right)^{2}}\left(\int_{1}^{r} F_{R}^{*}(r) \frac{d r}{r}+1\right)^{2},
\end{aligned}
$$


which shows just the validity of the former part of the theorem. The latter part is obtained by merely taking a special value $r=1$.

Remembering theorem 5, we may replace the second factor of the estimate in the inequality of theorem 8 by the square of the rational function $\Lambda(r)$ defined there; in fact, we have

$$
\int_{1}^{r} F_{R}^{*}(r) \frac{d r}{r}+1<\Lambda(r) \quad \text { for } 1<r<R .
$$

\section{Passage to limit.}

We can consider, instead of the family $\widetilde{F}_{R}=\{F(z)\}$, a related family $\mathbb{B}_{R}=\{G(z)\}$ defined by

$$
G(z)=\frac{1}{R} F(R z)=\sum_{n=-\infty}^{\infty} b_{n} z^{n} \quad\left(F(z) \in \mathfrak{\mho}_{R}\right) .
$$

Any member $w=G(z)$ of $\mathscr{G}_{R}$ maps $R^{-1}<z<1$ onto a finite (but not necessarily bounded) ring domain contained in $R^{-1}<w<\infty$ whose interior boundary component originated from $z=R^{-1}$ is $w=R^{-1} ; G(z)$ is further normalized by the condition $G\left(R^{-1}\right)=R^{-1}$. The Laurent coefficients of $G(z)=R^{-1} F(R z)$ are connected with those of $F(z)=\sum c_{n z} z^{n}$ by

$$
b_{n}=R^{n-1} c_{n} \quad(n=0, \pm 1, \pm 2, \cdots) .
$$

Consequently, any result on the family $\mathfrak{\mho}_{R}$ will be readily transferred into the corresponding one on the family $\mathbb{S}_{R}$, and vice versa, by means of the interrelation just explained. We shall now consider the limiting case as $R$ $\rightarrow \infty$.

It follows from theorem 3 that a Laurent coefficient $b_{n}$ with a non-positive suffix of any function $G(z) \in \mathbb{S}_{R}$ satisfies

$$
\left|b_{n}\right|<4 R^{2 n-2} \quad(n \leqq 0) .
$$

The bound of the last inequality tends very quickly to zero as $R \rightarrow \infty$, a fact which would be previously expected. On the other hand, theorem 5 implies for any $G(z) \in \mathbb{S}_{R}$ an estimation

$$
\begin{aligned}
b_{n} \mid<e\left(\frac{\left(R^{2}-1\right)^{2}}{R^{2}\left(R^{2}+1\right)} n-\frac{2 R^{3}-R^{2}-2 R-1}{R^{2}\left(R^{2}+1\right)}+\frac{2 R}{R^{2}+1} \frac{1}{n}\right) \\
\quad \text { for } n>\frac{1}{\lg R} .
\end{aligned}
$$

The bound of the last inequality tends to $e n$ as $R \rightarrow \infty$, so that there would follow the result of Littlewood [10], i. e.,

$$
f^{(n)}(0)<n ! e n
$$

that the equality sign may be rejected here would be readily seen from the proof of theorem 5 .

A rigorous proof of just mentioned fact can be performed as follows. Suppose that any function 


$$
f(z)=\sum_{n=1}^{\infty} a_{n} z^{n} \quad\left(a_{1}=1\right)
$$

regular and univalent in ' $z<1$ be given. For any $R>1$, Koebe's distortion theorem implies

$$
\left|f\left(R^{-1} e^{i \theta}\right)\right| \leqq \frac{R}{(R-1)^{2}} .
$$

Let $H(w)$ map the simply-connected domain consisting of the exterior of the curve defined by

$$
w=f\left(R^{-1} e^{i \theta}\right) \quad(0 \leqq \theta<2 \pi)
$$

onto the exterior of the circle with center at the origin and radius equal to $R^{-1}$ in such a manner that the points at infinity correpond to each other. By virtue of the principle of maximum modulus applied to $w / H(w)$, we can conclude that there holds

$$
\left|f(z) \leqq \frac{R^{2}}{(R-1)^{2}}\right| H(f(z)) \mid \quad\left(R^{-1} \leqq|z|<1\right) .
$$

Since the function $H(f(z))$ belongs to the family $\mathfrak{G}_{R}$, we get, for any $r$ with $R^{-1} \leqq r<1$,

$$
\begin{aligned}
\left|a_{n}\right| & \leqq \frac{1}{r^{n}} \frac{1}{2 \pi} \int_{0}^{2 \pi}\left|f\left(r e^{i \theta}\right)\right| d \theta \\
& \leqq \frac{1}{r^{n}} \frac{R^{2}}{(R-1)^{2}} \frac{1}{2 \pi} \int_{0}^{2 \pi}\left|H\left(f\left(r e^{i \theta}\right)\right)\right| d \theta \\
& \leqq \frac{1}{r^{n}} \frac{R^{2}}{(R-1)^{2}} \frac{1}{R}\left(\int_{R-1}^{r} F_{R}^{*}(R r) \frac{d r}{r}+1\right),
\end{aligned}
$$

the last inequality being a consequence of Prawitz's theorem; cf. the proof of theorem 1. Since, as noticed above, there holds a limit-equation

$$
\lim _{R \rightarrow \infty} \frac{F_{R}^{*}(R z)}{R}=\frac{z}{(1-z)^{2}}
$$

for $0<z<1$ uniformly in the wider sense, we get

$$
\left|a_{n}\right| \leqq-\frac{1}{r^{n}} \int_{0}^{r} \frac{1}{(1-r)^{2}} d r=\frac{1}{r^{n-1}(1-r)}
$$

valid for any $r$ with $0<r<1$, whence follows, by putting $r=e^{-1 /(n-1)}$, the aimed inequality

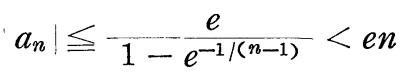

(it would be more preferable in the last step to put $r=1-1 / n$ ).

The argument just performed is essentially a mere reduction to the own analysis by Littlewood. However, the fact that the Littlewood's estimation may be regarded as a limiting form of our result can be proved also by 
means of a more straightforward consideration. In fact, for that purpose it is sufficient to verify that any function $f(z)$ under consideration can be approximated by a function $G(z) \in \mathbb{S}_{R}$ as a limit for $R \rightarrow \infty$. We shall prove here more precisely the following approximation theorem.

THEOREM 9. Let $f(z)$ be an analytic function regular and univalent in $|\boldsymbol{z}|<1$, normalized at the origin such as $f(0)=0$ and $f^{\prime}(0)=1$. Let the image of $|z|$ $<1$ by $w=f(z)$ be denoted by $\Delta$ and its boundary by $\Gamma$. For each positive number $R$ greater than unity, let a positive number $\rho(<1 / 4)$ be uniquely so defined that the ring domain bounded by $|w|=\rho$ and $\Gamma$ possesses the modulus equal to $\lg R$. Let further $K_{R}(z)$ denote the function which maps $R^{-1}<|z|<1$ onto this ring domain and satisfies the normalization $K_{R}\left(R^{-1}\right)=\rho$. Then the function defined by

$$
G_{R}(z)=\frac{1}{\rho R} K_{R}(z)
$$

belongs to the family $\mathbb{S}_{R}$ and satisfies the limit-equation

$$
\lim _{R \rightarrow \infty} G_{R}(z)=f(z)
$$

valid uniformly in the wider sense in $0<|z|<1$.

Proof. That the quantity $\rho=\rho(R)$ corresponds uniquely to $R$ is a consequence of the monotony character of moduli. It is evident from Grötzsch's distortion theorem that the family $\left\{G_{R}(z)\right\}(1<R<\infty)$ is normal in $0<z$ $<1$ (in the wider sense; for any $\delta$ with $0<\delta<1$, the function $G_{R}(z)$ is surely defined in $\delta<z^{<}<1$ provided $\left.R \geqq \delta^{-1}\right)$. Hence, it will suffice to prove that limit function $g(z)$ of any sequence $\left\{G_{R_{\nu}}(z)\right\}_{\nu=1}^{\infty}$ with increasing $R_{\nu} \rightarrow \infty$ chosen from the family and convergent uniformly in the wider sense in $0<z<1$ is always coincident with one and the same function $f(z)$. Let now the Laurent expansions of $G_{R_{\nu}}(z)$ and $g(z)$ be

$$
G_{R_{\nu}}(z)=\sum_{n=-\infty}^{\infty} B_{n \nu} z^{n} \quad\left(R_{\nu}{ }^{-1}<|z|<1\right)
$$

and

$$
g(z)=\sum_{n=-\infty}^{\infty} B_{n} z^{n} \quad(0<|z|<1)
$$

by assumption, we have

$$
\lim _{\nu \rightarrow \infty} B_{n \nu}=B_{n} \quad(n=0, \pm 1, \pm 2, \cdots) .
$$

Now, theorem 3 gives an estimation

$$
\left|B_{n \nu}\right|<4 R_{\nu}^{2 n-2} \quad(n \leqq 0)
$$

which implies

$$
B_{n}=0 \quad(n \leqq 0)
$$

and hence the origin being a removable singularity of $g(z)$, this function 
may be regarded as regular throughout ' $z<1$; evidently $g(0)=0$. On the other hand, theorem 5 gives an estimation

$$
B_{n v}<e n+o(1) \quad \text { as } R \rightarrow \infty
$$

valid uniformly with respect to $n$ and $\nu$, so that the relation of normalization

$$
1=R_{\nu} G_{R_{\nu}}\left(R_{\nu}^{-1}\right)=\sum_{n=-\infty}^{\infty} B_{n \nu} R_{\nu}^{-n+1}
$$

yields, by letting $\nu \rightarrow \infty$, an equation

$$
1=\lim _{\nu \rightarrow \infty} B_{1 v}=B_{1}=g^{\prime}(0) .
$$

Thus, $g(z)$ satisfies the same normalization as $f(z)$ at the origin. Since the sequence of the image-domains of $R_{\nu}^{-1}<z<1$ by $w=G_{R_{\nu}}(z)$ possesses the domain $\Delta$ pricked at the origin as its domain-kernel and converges to it in the sense of Carathéodory [4], the limit function $w=g(z)$ maps $0<z<1$ onto this pricked domain and hence maps the whole unit circle $z<1$ onto 4. This, together with the relations $g(0)=f(0)(=0)$ and $g^{\prime}(0)=f^{\prime}(0)(=1)$, implies the desired identity $g(z) \equiv f(z)$.

It is noted, by the way, that the quantity $\rho=\rho(R)$ introduced in the theorem satisfies, by virtue of Koebe's distortion theorem, the inequality

$$
\frac{R^{-1}}{\left(1+R^{-1}\right)^{2}} \leqq \rho \leqq \frac{R^{-1}}{\left(1-R^{-1}\right)^{2}} .
$$

Hence, the function $K_{R}(z)$ also tends to $f(z)$ as $R \rightarrow \infty$.

\section{REFERENCES}

[1] Bernardi, S. D., A survey of the development of the theory of schlicht functions. Duke Math. Journ. 19(1952), 263-287.

[2] Bieberbach, L., Über die Koeffizienten derjenigen Potenzreihen, welche eine schlichte Abbildung des Einheitskreises vermitteln. Sitzungsber. preuss. Akad. Wiss. Berlin (1916), 940-955.

[3] Bieberbach, L., Zwei Sätze über das Verhalten analytischer Funktionen in der Umgebung wesentlich singulärer Stellen. Math. Zeitschr. 2(1918), 158-170.

[4] Carathéodory, C., Untersuchungen über die konformen Abbildungen von festen und veränderlichen Gebieten. Math. Annalen 72(1912), 107-144.

[5] FABER, G., Neuer Beweis eines Koebe-Bieberbachschen Satzes über konforme Abbildung. Sitzungsber. bayer. Akad. Wiss. München (1916), 39-42.

[6] Garabedian, P.R., and M. Schiffer, A proof of the Bieberbach conjecture for the fourth coefficient. Journ. Rat. Mech. Analysis 4(1955), 427-465.

[7] Gronwall, T. H., Some remarks on conformal representation. Ann. of Math. (2) $16(1914 / 5), 72-76$.

[8] Grötzsch, H., Über einige Extremalprobleme der konformen Abbildung, II. Leipz. Ber. 80 (1928), 497-502.

[9] Komatu, Y., Untersuchungen über konforme Abbildung von zweifach zusammenhängenden Gebieten. Proc. Phys.-Math. Soc. Japan, 25(1943), 1-42.

[10] Littrewood, J.E., On inequalities in the theory of functions. Proc. London Math. Soc. $23(1925), 481-519$.

[11] Löwner, K., Untersuchungen über schlichte konforme Abbildung des Einheitskreises, I. Math. Annalen 89(1923), 103-121. 
[12] Nehari, Z., and B. Schwarz, On the coefficients of univalent Laurent series. Proc. Amer. Math. Soc. 5(1954), 212-217.

[13] Prawitz, H., Über Mittewerte analytischer Funktionen. Arkiv för Mat., Astr. och Fys. 20 A $(1927 / 8), 1-12$.

[14] Schaeffer, A.C., ANd D. C. Spencer, Coefficient regions for schlicht functions. New York (1950). xv + $311 \mathrm{pp}$.

[15] TeichмülleR, C., Untersuchungen über konforme und quasikonforme Abbildung. Deutsche Math. 3(1938), 621-678.

Department of Mathematics,

Tokyo Institute of Technology. 